\title{
Proprioceptive pathways of the spinal cord
}

\author{
RICHARD J. SCHNEIDER, A. T. KULICS, AND THOMAS B. DUCKER \\ From the Division of Neurological Surgery, University of Maryland School of Medicine, Baltimore, \\ Maryland, USA
}

S U M M ARY In the Macaque, surgical lesions were made in the dorsal funiculus, in the dorsolateral funiculus, and through half of the spinal cord. The somatosensory and motor capacity of the animal were examined neurologically and electrophysiologically. The exact lesion was then confirmed pathologically in detail. The results of these experiments indicate that limb position information from the distal limb and proximal limb are relayed to the brain in two different fashions. Distal limb position information, especially the cortical representation of the limbs' volar surface as it moves in space, is drastically impaired by dorsal funiculus or posterior white column lesions. Proximal limb position may or may not be impaired by similar lesions, for this information while initially in the dorsal or posterior white columns is sorted out (as it ascends in the spinal cord) to the dorsolateral funiculus or white columns. For example, in the lower thoracic spinal cord, both distal and proximal hind limb sensation are impaired by posterior white column damage; in the cervical cord, only distal sensation is impaired by the same lesion, and proximal information is spared. We refer to this neuroanatomic rearranging as 'fibre sorting', and we believe that it is clinically significant in spinal cord disease.

The role of the dorsal funiculus or the posterior white columns of the spinal cord in somatic sensibility has for some time been an issue of debate. Dissatisfaction has arisen with the accepted and widely disseminated viewpoint, as stated by Mountcastle and Darian-Smith (1968): 'What remains in the mechanoreceptive sphere after large fiber or dorsal column lesions is the capacity to recognize that a mechanical stimulus has occurred; it is no longer possible to specify it as to intensity, location or shape.' This interpretation is supported by much of the clinical literature. The results of neurological examinations of humans with various spinal cord pathologies are often interpreted as Walshe (1970) does: 'The . . . sensory afferent path which subserves touch and pressure, postural sense and also vibration sense turns upwards in the dorsal columns and reaches the dorsal column nucleus of the medulla on the side of entry.' Though recognition has been given by Walshe to the fact that the dissociation of func-

This work was initiated at the University of Pittsburgh with NIH grants MH11682 and NB07712, and completed at the University of Maryland with funds from the Bressler Foundation.

Address for reprint requests: Dr R. J. Schneider, Neurosurgery Division, 12th Floor, University of Maryland Hospital, Baltimore, Maryland 21201, USA.

Accepted 15 November 1976 tion between the dorsal funiculus and the lateral funiculus is not so clear-cut as diagrams picture it, the general interpretation accepts this dissociation as fundamental.

Animal experiments have not given such clear results. Thus Ferraro and Barrera (1934) found that macaques with posterior or dorsal funicular lesions rostral to the cervical plexus showed few deficits in the ability to use their hindlimbs. They attributed this to the preservation of proprioceptive sensation fibres from the hindlimb ascending in the spinocerebellar tracts in the lateral funiculus, and thus escaping their lesions. Similar results from a series of experiments on macaques done by Gilman and Denny-Brown (1966) led them to hypothesise that lesions of the posterior white columns produced a deficit in the ability to explore and orient to extrapersonal space, but spared manipulation like grooming which involved intrapersonal space. Moreover, electrophysiological and anatomical studies have shown that somatosensory information can, under certain conditions, be transmitted by other than lemniscal pathways (Albe-Fessard, 1967), and that pathways other than those in the dorsal funiculus may contribute more heavily to somatosensation in certain species such as carnivores (Bowsher, 1965). 
These results indicate a more limited role for the funiculi gracilis and cuneatus. Semmes (1969), in re-examining the concepts of epicritic and protopathic sensation, called attention to the possibility that the phylogenetically newer 'lemniscal system' was specially involved in exploration and manipulation or active touch. Wall (1970) concluded that the dorsal funiculi were necessary for external search behaviour when internal search for identification of a somatosensory event failed to produce a satisfactory answer. He wrote: 'Impulses ascending in the dorsal column-medial lemniscus system are involved in controlling the analysis of messages arriving over the other somatosensory pathways.' This restricted the role of the dorsal funiculi to that of a governor in the cybernetic sense. Melzack and Bridges (1971), after a series of experiments on cats with dorsal funiculus lesions, augmented Wall's control system view by concluding that the dorsal funiculi played an important role in the choice of an appropriate behaviour programme. Dubrovsky et al. (1971), delimited this role in behavioural programme selection by concluding, from their work on cats, that the dorsal funiculus was necessary for planning and execution of serial acts in which accurate timing, precise forelimb projection, and space orientation are necessary conditions.

Concurrent with this behavioural research, a body of anatomical and electrophysiological data was growing which supplemented and modified traditional concepts of the posterior white column. Thus, it was demonstrated that only $25 \%$ of the myelinated fibres entering these columns from the hindlimbs reach the gracilis nuclei (Glees and Soler, 1951). Analyses of peripheral afferent fibres (Iggo, 1963) showed that in several different types mechanoreceptive information was being transmitted centrally. Later electrophysiological experiments showed that fibres conveying several of these modes of mechanoreceptive information present at more caudal levels of the dorsal white columns disappeared, or were severely diminished, at more rostral levels in cats (Petit and Burgess, 1968; Burgess and Clark, 1969) and in monkeys (Whitsel et al., 1969). Furthermore, a segregation of submodalities was seen in the nuclei of the dorsal funiculus between proprioceptive and exteroceptive afferent information (Kuhn, 1949; Gordon and Paine, 1960; Gordon and Seed, 1961; Perl et al., 1962; Gordon and Jukes, 1964; Biedenbach, 1972), and this dissociation of submodalities was reflected by the cell types in these regions (Kuypers and Tuerk, 1964; Hand, 1966; Biedenbach, 1972). A similar dissociation has been shown at thalamic (Jasper and Bertrand, 1964; Mallart,
1964; Andersson et al., 1965; Landgren et al., 1965; Albe-Fessard, 1967) and cortical levels (Powell and Mountcastle, 1959b; Schneider, 1972; Dreyer et al., 1974).

In the light of these various lines of experimental results, we proposed to determine whether it is necessary to postulate a special role for the dorsal white column in somatic sensibility in order to explain the existing behavioural and neurological evidence, or whether a reinterpretation of previous results considering such processes as fibre sorting could explain these findings.

\section{Methods}

Preoperative and postoperative neurological and behavioural examinations were performed on each of 18 adolescent monkeys (Macaca mulatta) which were confined in a restraint apparatus (Carlson, 1972). Preoperatively, the examinations were carried out from one to several hours before the surgical lesions. The surgical procedure was attendant upon completion of a full examination without questionable responses in any category. Postoperatively, several examinations were made at different periods of elapsed time from the surgical procedure-usually within the first week, and again on the second and fourth weeks. Behavioural observations were also made on the animals preoperatively and postoperatively, with special attention to the immediate postoperative period.

The neurological examinations tested the following six categories of response: (1) grasp reactions-that is, the true grasp reflex and instinctive grasping-which are distinguished by the fact that in ascending order of complexity lie the coarse grasp or traction reaction, the true grasp reflex, and instinctive grasping (Denny-Brown and Chambers, 1958; Twitchell, 1965); this lastnamed reaction is a contactual orienting reaction whose voluntary performance has also been called spontaneous grip or spontaneous grasp (Ferraro and Barrera, 1934); (2) tactile and contact placing (reflexes of the hindlimb); (3) limb reactions to light touch of the skin, brushing of the hairs, pricking and pinching (with pin and toothed forceps); (4) muscle tonus; (5) proprioceptive reactions-ability to coordinate limb movements in space, to locate and secure extrapersonal objects, and to maintain normal as opposed to catatonic postures; (6) plantar and patellar tendon reflexes. Forelimb tactile placing was not reported in our experimental results because the various lesions did not offer us the chance to evaluate enough animals where fibre sorting in the fasciculus cuneatus could have occurred. 
The standard neurological tests were undertaken especially to evaluate any damage to motor pathways in the lateral funiculus which might influence our behavioural observations or electrophysiological recording. We felt that corticospinal tract damage would have implied damage to the dorsal part of the lateral funiculus which would also have interrupted somatosensory projections other than the dorsal white columns, such as the dorsal spino-cerebellar tract, and Morin's pathway (Ha and Morin, 1964; Ha, 1971), the spino-cervicolemniscal tract. Similarly, the integrity of the anterolateral projection was assessed by pricking and pinching stimuli, since damage here would imply ventral spinocerebellar tract involvement as well as interruption of mechanoreceptive afferent fibres within the anterolateral pathway.

The operative procedure was as follows. Animals were anaesthetised with sodium pentobarbitone $30 \mathrm{mg} / \mathrm{kg}$ ip (five animals) or $25 \mathrm{mg} / \mathrm{kg}$ iv (13 animals). Intravenous catheters were inserted for supplemental anaesthesia during surgery. A laminectomy was then performed aseptically. The dura mater was split and retracted with suture ties under a microscope; the pia mater was also retracted. A Bard-Parker gauge 11 blade, cut off, marked in millimetre gradations, and held in a haemostat was used to make the lesion. The dura mater was then reapposed with 5-0 gut or silk, gel-foam was placed over the cut spinous processes, and muscle, fascia, and skin rejoined. Procaine penicillin (one million units) was administered immediately after the operation, and for several days thereafter. Animals were observed after surgery, and any sign of infection was treated promptly. Three control laminectomies were performed using the same procedures; permanent neurological defects and electrophysiological changes were absent from these preparations.

Microelectrode recordings were obtained from the primary somato-sensory cortex of the monkeys approximately one month after spinal surgery (Schneider, 1972; Dreyer et al., 1974). At the end of each electrophysiological experiment, the animal was deeply anaesthetised with pentobarbitone, and sacrificed by intracardial infusion with $0.9 \%$ saline followed by $10 \%$ neutral buffered formalin. Appropriate portions of the brain were imbedded in celloidin, and coronal or sagittal sections were taken at $30 \mu \mathrm{m}$; the sections were stained with cresylecht violet. The spinal cord in the area of the lesion, as well as selected sections rostral and caudal to it, were also imbedded in celloidin. The extent of the lesion was determined by examining Mahon stained sections, while anterograde and retrograde tract degeneration were determined by the Marchi method. Reconstructions of spinal cord lesions were drawn from slide projections of histological sections. These are displayed in Figs 1, 2, and 3. Cortical microelectrode penetrations were reconstructed to permit analysis of the cytoarchitectural area traversed and laminar location of the neurones isolated in each penetration (Schneider, 1972; Dreyer et al., 1974).

\section{Results}

Basically, there are four spinal cord lesions: the dorsal funiculus, the lateral funiculus (in the dorsal aspect only), the dorsal and dorsolateral funiculus combination, and the combination lesion extended into the cord hemisection. The dorsal funicular lesion cut the posterior white tracts, funiculus gracilis in the low thoracic area, and both gracilis and cuneatus above. The lateral funicular lesion spared the motor tracts and the anterolateral quadrant but attempted to cut the posterior spinocerebellar tracts and Morin's pathway. The dorsal and dorsolateral lesion (see Fig. 4) included all those tracts to various extents. Finally, the hemisection definitely included the motor system.

\section{LESIONS OF THE DORSAL FUNICULUS}

Perhaps the signal manifestation of an animal with a complete dorsal white matter lesion is his propensity to place the most distal aspect of the extremity (hand or foot) on the floor with its dorsal rather than ventral surface down. We call this dorsal placing. This observation has been emphasised by others (Ferraro and Barrera, 1934; Gilman and Denny-Brown, 1966). It occurred even when the limb was oriented appropriately, and it was most noticeable in animals with only a unilateral lesion, but it was definitely present in bilateral lesions.

Lesions in the dorsal funiculus alone were either in the thoracic area or cervical area. When the animal had a low thoracic lesion, both distal and proximal proprioceptive function were markedly impaired, and the animal had little use of the lower limb. In high thoracic lesions, the monkey demonstrated normal posture and a steady gait. It would be active and climb the cage wall. But in the feet, grasp was impaired, palpatory behaviour was diminished, dorsal placing of the foot did occur, and distal malalignment was noticeable. Thus, distal proprioceptive function was impaired but proximal proprioception appeared spared. In midcervical lesions, the same 


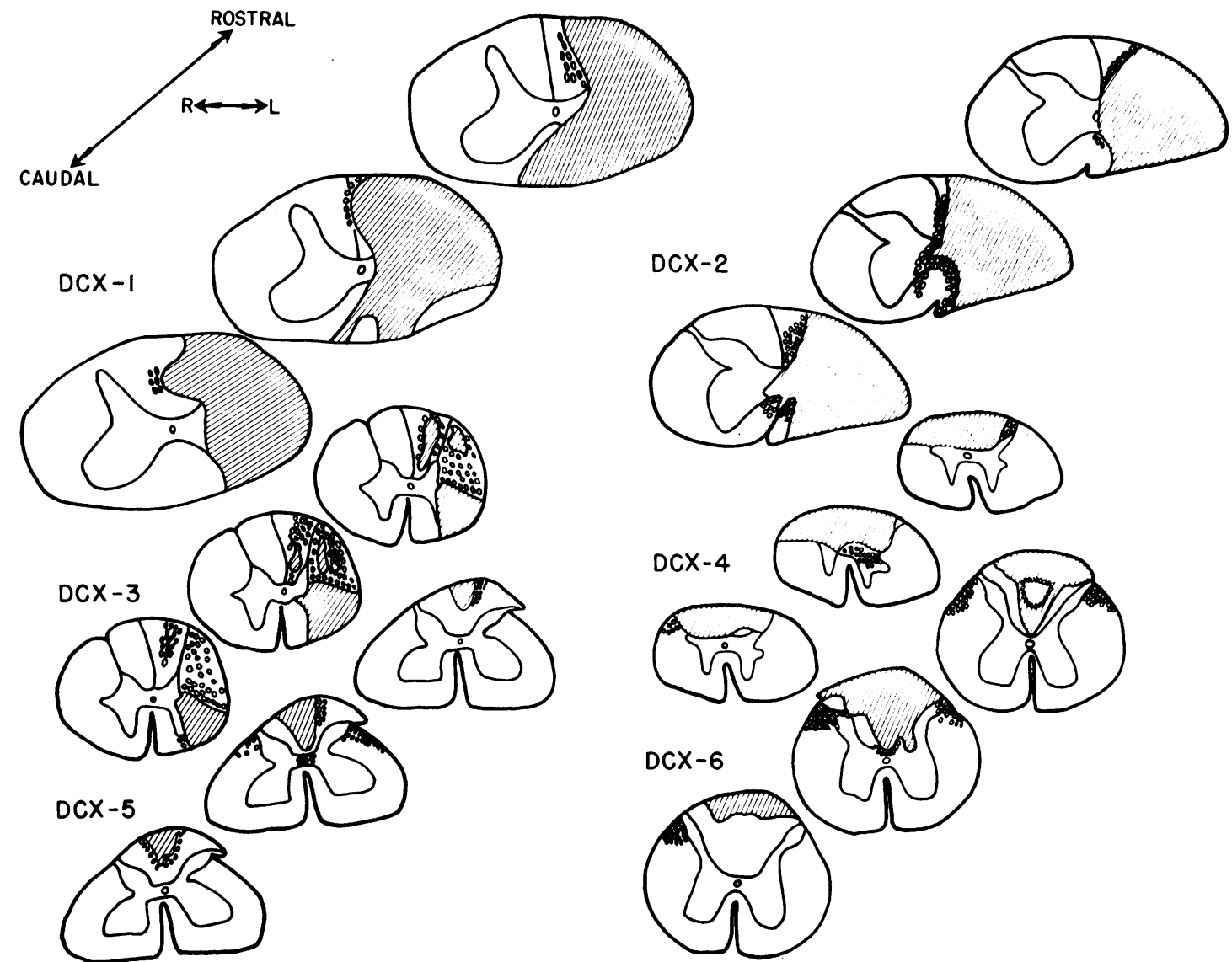

Fig. 1

Figs. 1, 2, and 3 Drawings made from projections of stained tissue sections from each animal in this experimental series. Total extent of lesions was determined by overlapping consecutive sections. Striped area implies total damage, circles imply partial damage. Rostral and caudal drawings were made at levels beyond which lesion size diminished with no additional pathology. Rostral drawings in DCX:12 were determined by ascending degeneration pattern, only two drawings were done for $D C X: 8$, and $D C X: 15$ was drawn from written account of total extent of lesion.

deficit was present in the feet and, in addition, the forelimbs were now markedly affected. The arms flailed during ambulation, and were held in a sling-like posture at rest. The animal would use the entire forearm to palpate the surroundings. There was some recovery with time but skilled manipulation was dependent on visual cues. In the very high cervical lesions in one animal forelimb, proximal proprioceptive function appeared to recover, but distal position and stereopsis remained disordered.

\section{I.FSIONS OF THE LATERAL FUNICULUS}

In lateral funicular lesions, in the dorsolateral (posterolateral) part of the cord, behind the dentate ligaments, with sparing of the corticospinal tracts, the animals had surprising difficulty. In both low cervical lesions, the animals upon recovery from surgery would climb their cage shelf and cage walls, but showed a preference for lying on the cage floor, with hindquarters slightly elevated because of an increased tonus in the hip extensors. The limbs ipsilateral to the lesion had no trouble localising objects in space, but upori reaching for such objects their grasp was markedly diminished in vigour. The orientation of their feet at rest was often at unusual rotational angles to the body-that is, at right angles to the direction the body was facing. Forelimbs most often showed a posture of shoulder adduction, 

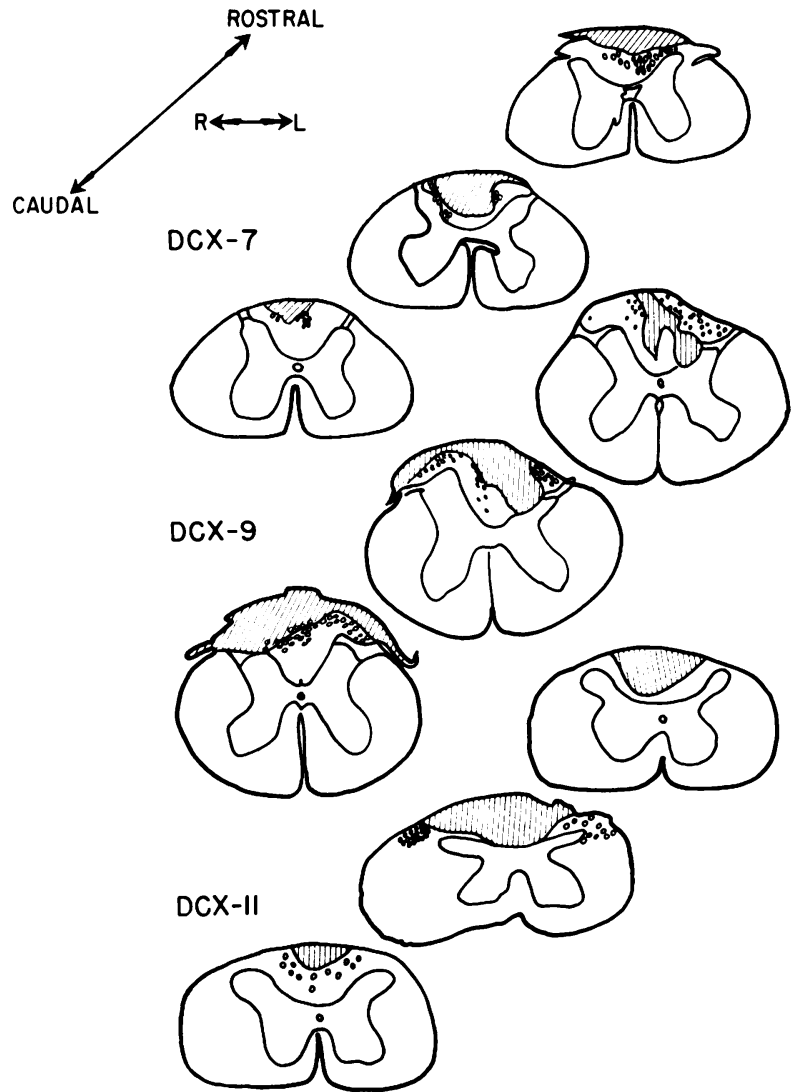

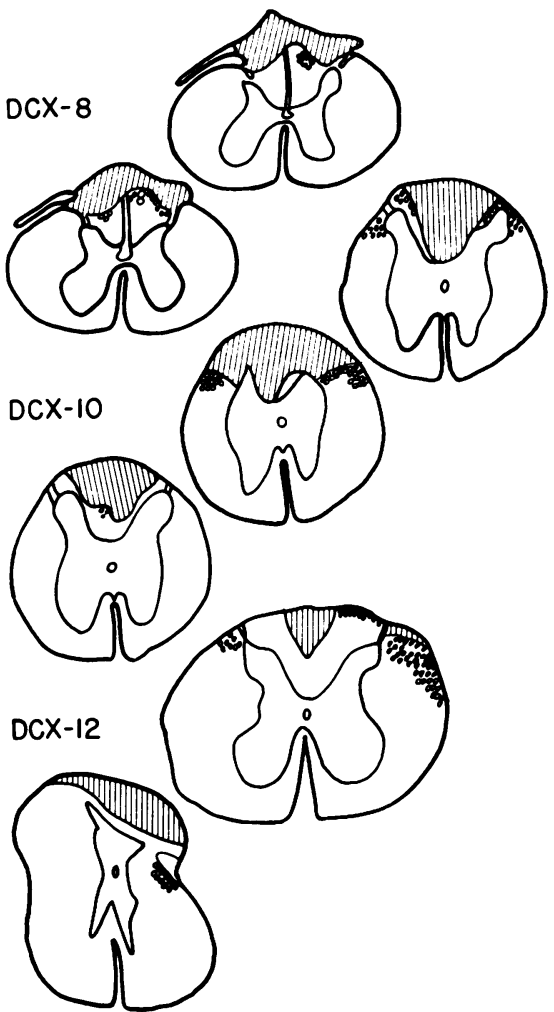

Fig. 2

elbow and wrist flexion when the animals were in a sitting position, somewhat like the animals with high dorsal funiculus lesions. The affected hindlimbs manifested a slight resting tremor, and showed plantar flexion of the toes at rest, which was greater at the interphalangeal than at the metatarso-phalangeal joints. During ambulation the toes tended to be widely splayed providing a greater contactual circumference. With vision diverted, distal limb contact with objects caused peculiar reactions. Among these were staring in the direction of the surface touched, repeated collision with the surface when vision remained elsewhere directed, and what may have been a startle reaction to encountering the object.

\section{MORE EXTENSIVE LESIONS}

Combining lesions of both dorsal and dorsolateral funiculi from the sixth cervical to the seventh thoracic segment had a profound effect on the animal, for it made no attempt at movement in and around the cage. The monkeys refused to stand and would lie in postural hyperextension (Gilman and Denny-Brown, 1966). When the lesion was further extended to cord hemisection, there was additional flaccid paralysis of the ipsilateral extremity plus self-mutilation of the affected limb.

Tables 1 and 2 give the results of the neurological examinations done on animals whose tractotomies included pathways as verified by histology. The scheme of these tables includes only residual changes at one month after surgery since this was the time when electrophysiological mapping was commonly performed. Except with pinch (which was to study pain sensation), entries refer to the limb ipsilateral to the lesion.

To make the tables more understandable, follow a typical entry, DCX: 4. This was an animal with a lesion of the dorsal funiculus of the left side (see comment), at the level of the third thoracic segment of the spinal cord. One month after surgery, his left forelimb pulled away from grasp eliciting stimuli, and his left hindlimb failed to 


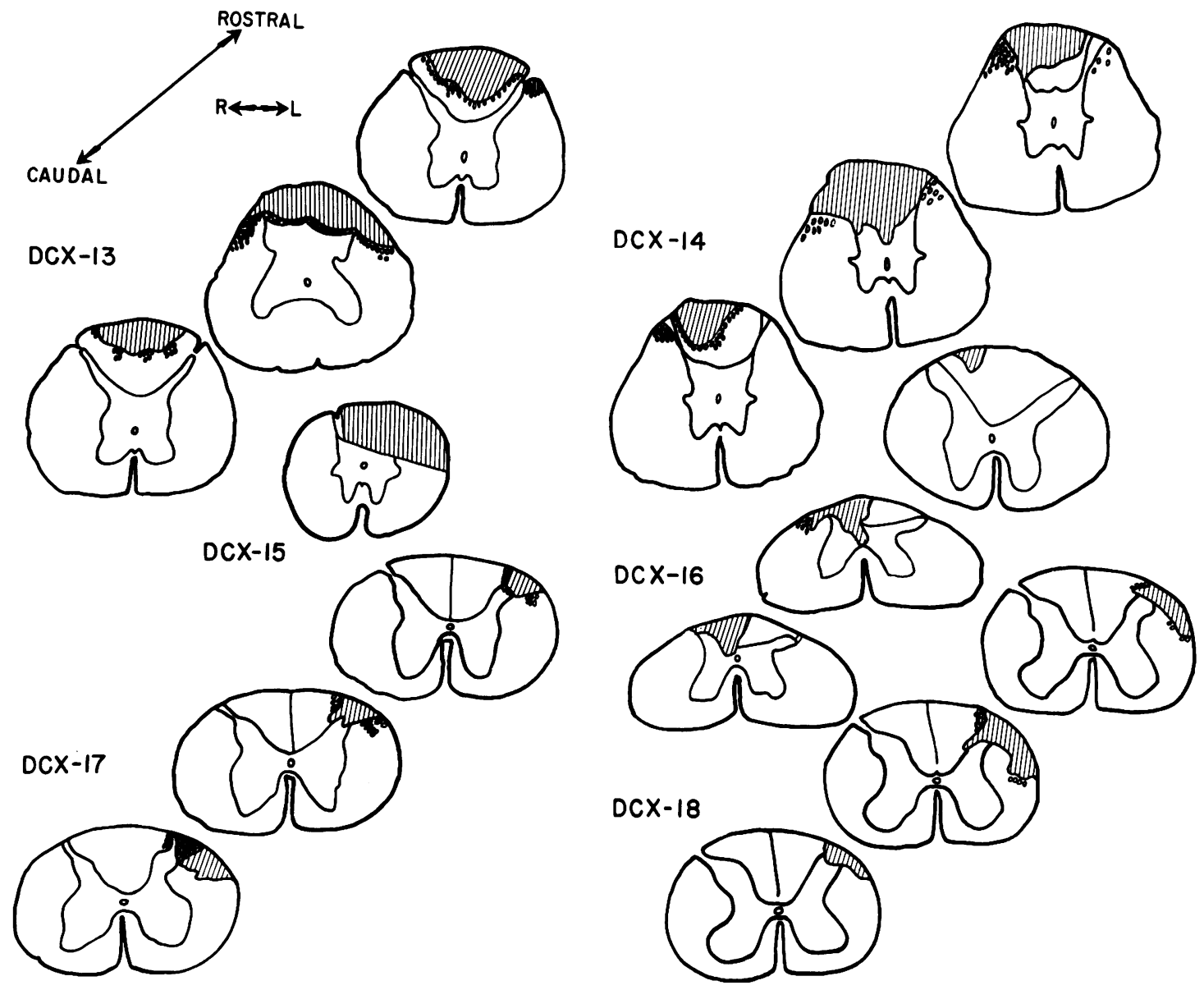

Fig. 3

react to such stimuli. He would palpate and orient to things with his left forelimb but not with his left hindlimb. The left hindlimb did not place when stimulation was delivered to hairs or lightly to the skin surface, but did place when deeper pressure to the foot was applied. The left forelimb but not the hindlimb reacted to hair displacement and light tapping on the skin surface. There was a normal withdrawal to pinch by the right limbs, normal muscle tone in the left limbs, normal limb movements on the left side when reaching for objects or orienting in space, and a normal patellar but no plantar reflex in the left hindlimb. The animal had a tractotomy which extended to the right side where it included the dorsal and dorsolateral funiculi. It is thus listed in Table 2 as well. (The comments column lists the side examined when both sides were so used, or when ambiguity might exist.)
Several of these results should be emphasised. The hindlimb grasp reflex is almost uniformly absent after dorsal funicular transection and after the combination lesion, with or without motor involvement, when above the level of entry for afferent fibres from the affected limb. Instead, the hindlimbs usually display no reaction, and the forelimbs show tactile avoiding, a response which appears in the early postoperative period in the hindlimbs, and diminishes thereafter. Partial dorsal funicular lesions (DCX: 8, DCX: 9, DCX: 15) may or may not spare some grasp reflex. In pure seventh cervical dorsolateral transections, the forelimbs were unaffected, while the hindlimbs could show a diminution which might be traceable to the weakened grasp rather than an absence of the reflex. In order to abolish forelimb instinctive grasping, most of the forelimb plexus should be below a dorsal funicular lesion or hemisection. 


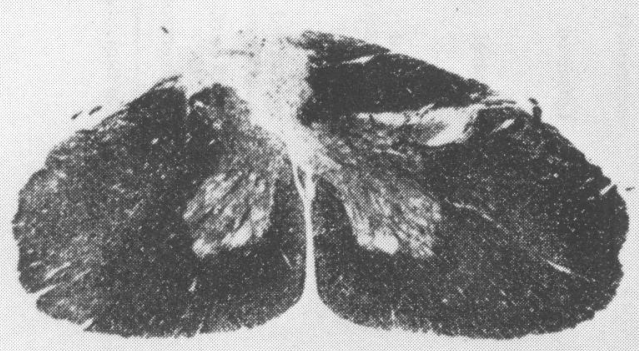

A
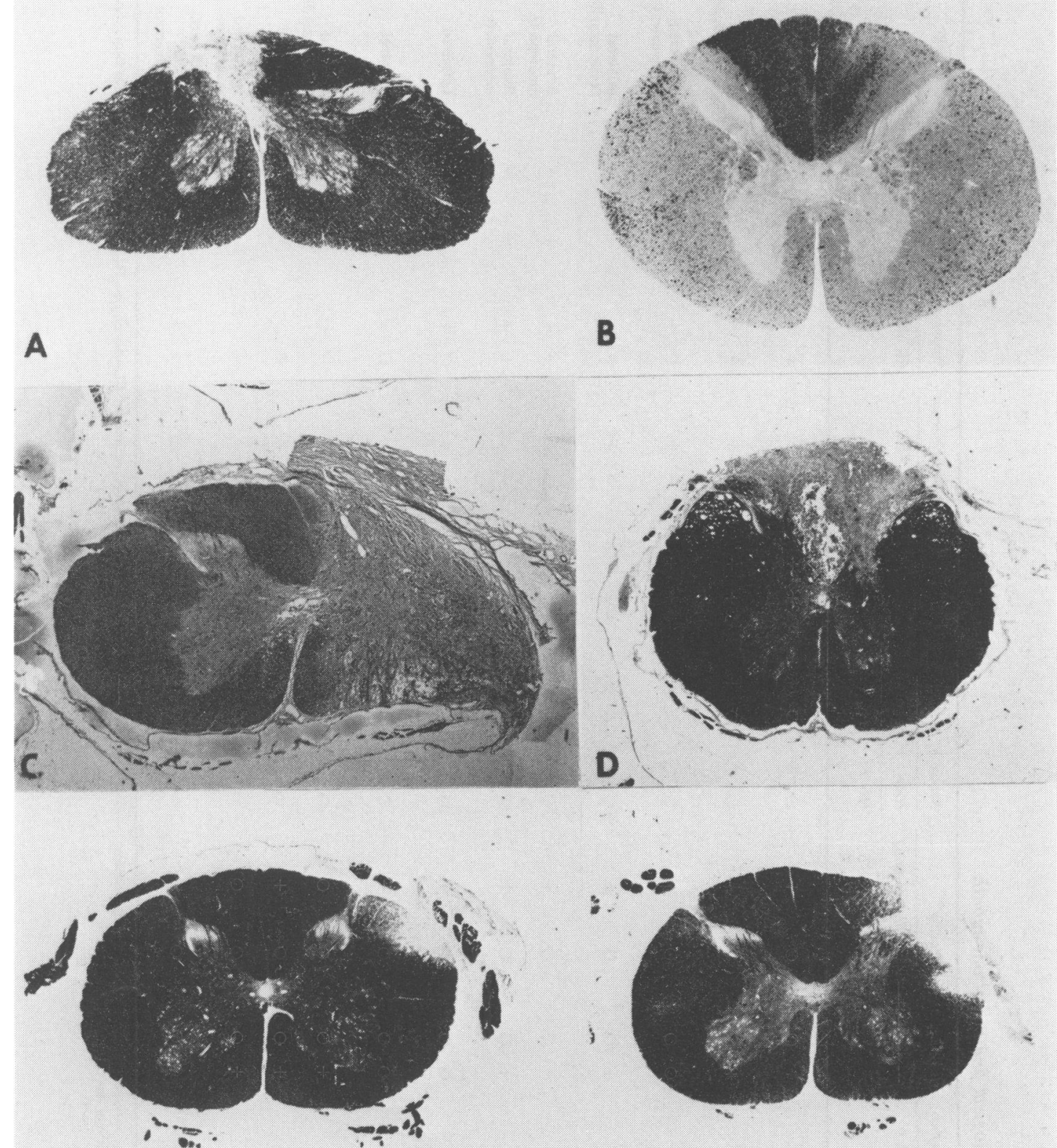

E

$\mathbf{F}$

Fig. 4 A. Complete right dorsal funiculus lesion at C5 segment with left dorsal funiculus involvement (DCX:16). B. Ascending pattern of axonal degeneration (DCX:16). C. Spinal cord hemisection at C7 segment, with partial sparing in medial regions of dorsal and ventral funiculi $(D C X: 2)$. D. Dorsal funiculus lesion at C3 segment with some dorsolateral funiculus involvement (DCX:6). E. Dorsolateral funiculus lesion at C7 segment involving mainly proposed area of projection of spino-cervico-lemniscal tract fibres with some sparing of dorsal spinocerebellar tract fibres (DCX:17) (see Fig. 3). F. Dorsolateral funiculus lesion involving spino-cervico-lemniscal tract and presumably most dorsal spinocerebellar tract fibres $(D C X: 18)$. 


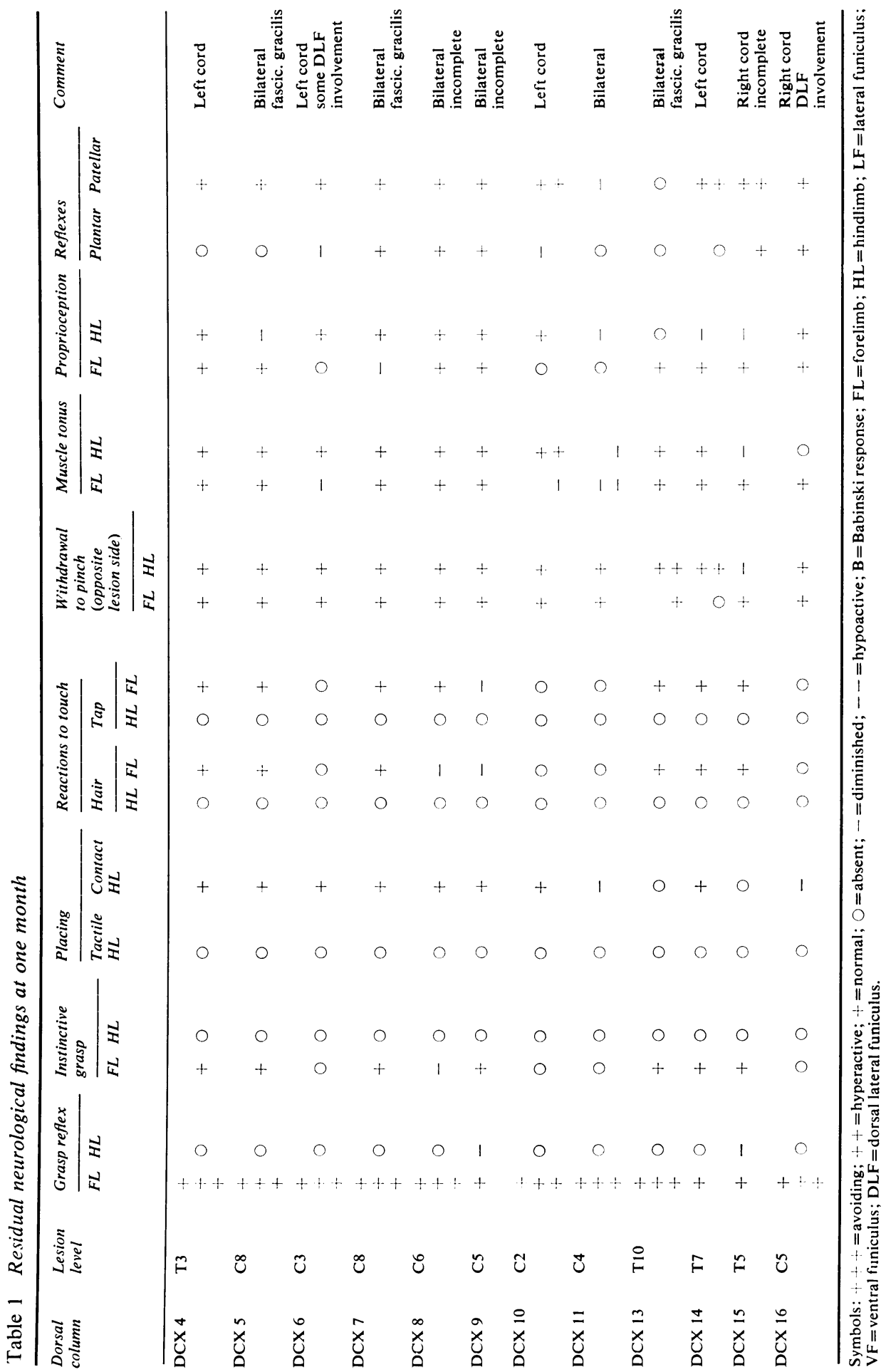




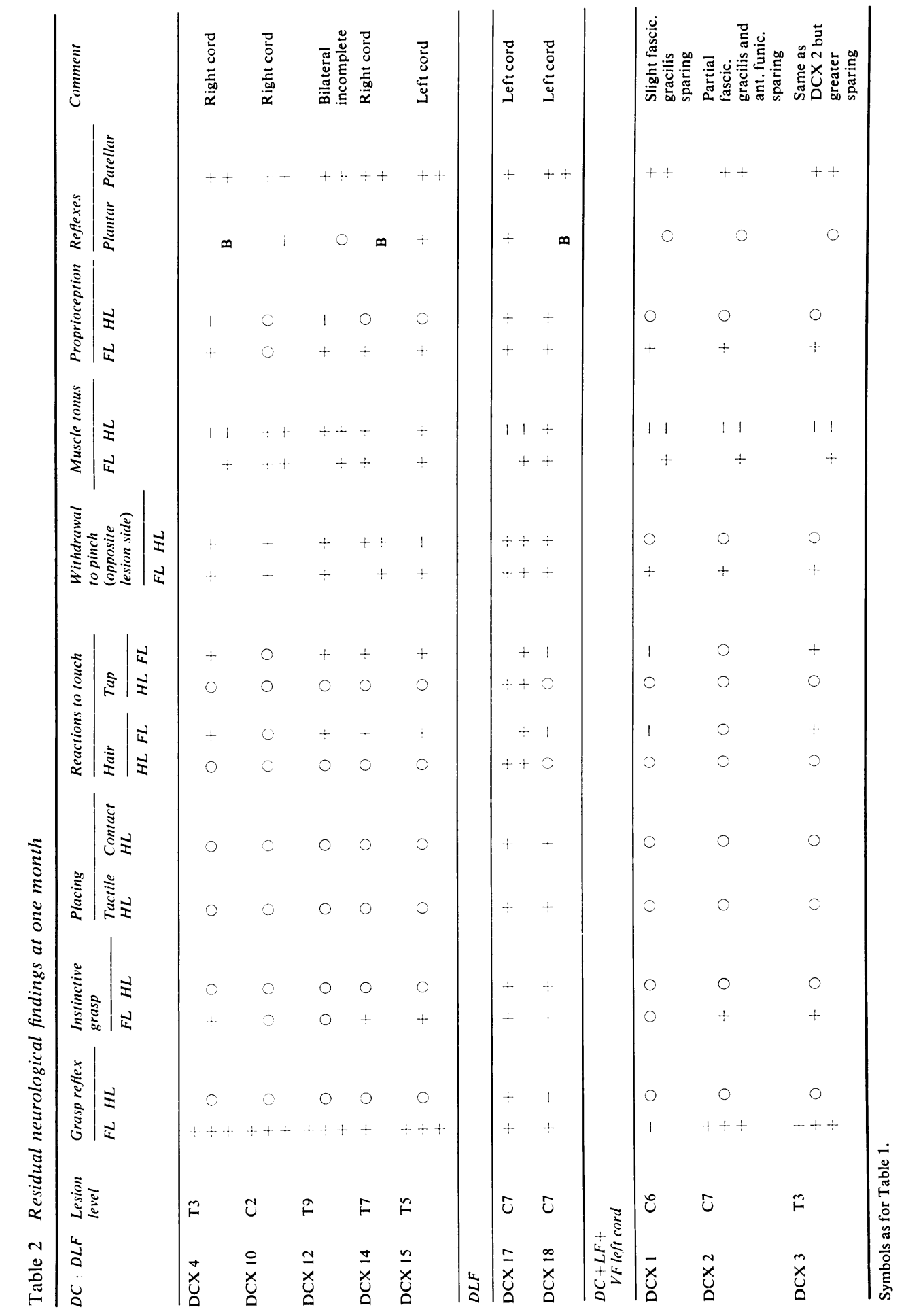


Thus, a fourth cervical lesion (DCX: 11), but not an eighth cervical lesion (DCX: 5, DCX: 7), abolishes forelimb instinctive grasping, and a sixth cervical hemisection (DCX: 1) leads to abolition, while a partial lesion at the fifth or sixth cervical segment (DCX: 8, DCX: 9) may or may not spare instinctive grasping in the forelimb. In the hindlimb, a pure dorsal funicular lesion at high thoracic to cervical levels will suffice to eliminate instinctive grasping, as does expanding the lesion to include additional pathology. But a seventh cervical dorsolateral lesion alone does not affect instinctive grasp in any limb (DCX: 17, 18).

Tactile, but not contact, placing of the hindlimb is abolished by pure posterior white column lesions, except in the cases of DCX: 13 which was an unusually low lesion, and DCX: 15 which had some postoperative complications affecting the legs. Both hindlimb tactile and contact placing disappeared with combination lesions, but were intact with pure dorsolateral lesions. Hair displacement reactions as well as light touch or tap reactions disappear pari passu with interruption of posterior white column fibres related to receptive fields on the body surface in question. Withdrawal to pinching is exhibited only in the contralateral limb whose fibres enter caudal to hemisection. After a month, muscle tone is seldom affected in pure posterior white column transected preparations, but hypotonia ensues with hemisections. Proprioception is completely absent in the forelimb after either posterior white column or a combination lesion above the brachial plexus. Proprioception is absent in the hindlimbs only when the posterior white column lesion is just above the lumbar enlargement, or when the lesion includes the lateral funiculus at higher segmental levels.

\section{Discussion}

We interpret our results to imply that the rearrangement of sensory afferent fibres, which has been shown to occur anatomically and electrophysiologically (Glees and Soler, 1951; Petit and Burgess, 1968; Burgess and Clark, 1969; Whitsel et al., 1969), produces predictable behavioural and neurological changes in the effects of tractotomies. The main outlines of this rearrangement, which we call 'fibre sorting', are (1) the redistribution of proprioceptive information from the hindlimb to pathways other than the dorsal funiculus as this information is transmitted rostrally, (2) the appearance of this proprioceptive information in the lateral funiculus (dorsal aspect) at rostral spinal cord levels, and (3) the rostral reorganisa- tion of exteroceptive information combined with the proprioceptive redistribution which concentrates, within the posterior white columns, afferent fibres related to hair displacement and those related to light touch on the glabrous skin surfaces of the distal limbs. We believe our results support this scheme in the following ways.

The contact (proprioceptive) placing reflex in the hindlimb is not abolished in macaques whose dorsal funiculus is interrupted at levels above T5 segment (DCX: 4, 5, 6, 7, 8, 9, 10, 11, 16), but is absent in macaques with dorsal funiculus interruptions at or below T5 (DCX: 13, 15), and in macaques with lateral funiculus or hemicord involvement above T4 segment (DCX: 1, 2, 3, 4, 10). However, lateral funiculus tractotomies alone (DCX: 17, 18) do not abolish this reflex. The contact placing reflex depends upon proprioceptive information being conveyed to the frontomotor cortex (Woolsey and Bard, 1963). Since transecting the dorsal funiculus does not necessarily abolish this reflex, the implication is that the necessary afferent proprioceptive information does not project in an uninterrupted manner exclusively through the posterior white column. In contrast, hindlimb tactile (exteroceptive) placing, a reflex depending on exteroceptive information being relayed to the parietal lobes (Woolsey and Bard, 1963), is abolished by dorsal funiculus lesions regardless of the level, and is not abolished by isolated lateral funiculus lesions. This implies that the necessary afferent exteroceptive information for the tactile placing reflex does project in an uninterrupted fashion exclusively through the posterior white column. The fact that focal lateral funiculus lesions which are dorsally placed do not abolish proprioceptive placing may imply a shared projection of these afferent fibres between the two tracts. But a more parsimonious conclusion would be that, since tactile placing is still intact in macaques with isolated lateral tractotomies, skin contact before the proprioceptive stimulus threshold is reached elicits a tactile placing reflex which obscures the fact that proprioceptive placing is absent.

Placing reactions have been examined after transections of the dorsal funiculus of macaques (Ferraro and Barrera, 1934; Gilman and DennyBrown, 1966; Liebman and Levitt, 1973), and in domestic cats (Lundberg and Norrsell, 1960; Dobry and Casey, 1972). In cats it was found that tactile placing disappeared after ipsilateral transection of the medio-dorsal lateral funiculus rostral to the level of the hindlimb afferent entry of the spino-cervico-lemniscal tract (Morin's pathway), and caudal to the lateral cervical 
nucleus (Lundberg and Norrsell, 1960). However, lesions of Morin's pathway in its contralateral ventral funicular region, cranial to the lateral cervical nucleus, do not abolish tactile placing in cats (Norrsell and Voorhoeve, 1962). It was concluded that Morin's pathway is not the exclusive afferent link in this cortical reflex for cats (Norrsell and Voorhoeve, 1962). Lesions of the dorsal funiculus at the first cervical level which included more than $90 \%$ of its afferent fibres, were also reported to abolish tactile placing in cats (Dobry and Casey, 1972).

In macaques, Gilman and Denny-Brown (1966) reported that to demonstrate tactile placing in the hindlimbs after fourth cervical dorsal funiculus transection requires brisk cutaneous contact to proximal parts of the leg, and in four of their animals (DC2, DC4, DC15, and DC46) they considered tactile placing to be absent. It was also found that the more extensive combination (dorsal and dorsolateral funiculus) lesion at the third cervical level was required to abolish contact placing (since these authors did not use contact placing, we make this assessment from their statement, 'no limb placed with even broad, heavy, prolonged contact') along with tactile placing in the hindlimb, and that dorsolateral funicular lesions alone left no residual defect in tactile placing (Gilman and Denny-Brown, 1966). In support of the notion that both dorsal funiculus and dorsolateral funiculus share fibres involved in the projection of proprioceptive information necessary to the contact placing reflex, other research indicates that this is the case for position sense (Vierck, 1966). The same studies infer that the anterolateral funiculus also pays a role in position sense.

We believe that the proprioceptive disturbances we observe are linked to afferent fibres which travel both in the dorsal funiculus and in pathways in the dorsolateral funiculus at more rostral levels. This may account for the occurrence of these disturbances in the hindlimbs of animals with low cervical and high thoracic, dorsal funiculus plus lateral funiculus tractotomies (DCX: 1, $2,3,4,10,14,15)$; in the forelimbs of animals with high cervical (DCX: 6, 10, 11) and the hindlimbs of animals with low thoracic (DCX:13) dorsal funicular tractotomies (in these, proprioceptive fibres have not yet undergone fibre sorting, although, a decrease in hindlimb position sense following a dorsal funiculus lesion as high as the seventh thoracic level has been reported (Ferraro and Barrera, 1934) and we observed one (DCX: 15 at the fifth thoracic level) but not in the forelimbs or hindlimbs of animals with dorsolateral funicular tractotomies at low cervical levels (DCX:17, 18). Animals occasionally showed proprioceptive dysfunctions which did not fit this systematic pattern (DCX: 5, 11), but we believe these are the exception rather than the rule.

Electrophysiological results in the isolated dorsolateral funiculus tractotomy experiments lend support to the occurrence of proprioceptive (deep-submodality) afferent fibres in the rostral dorsolateral funiculus. These data show that damage involving Morin's pathway does not affect the distal, deep-submodality, single cell representation in Brodmann's area 2 of macaques as severely as when the spinal cord position of the dorsal spinocerebellar tract is encroached upon (Schneider, 1972; Dreyer et al., 1974). This may imply simply that there is a direct relationship between the extent of a dorsolateral funiculus lesion, and the loss of afferent information projecting to cytoarchitectural area 2 of the brain. This evidence tends to allocate a role, if not the exclusive role, in relaying deep afferent impulses to the primate dorsal spinocerebellar tract. However, the evidence is that the contact placing reflex is mediated through the frontomotor areas in macaques, so that change in occurrence of proprioceptive units in Brodmann's area 2, while significant for somatic sensation, would not account for the abolition of this response. Recent results (Asanuma and Rosén, 1972a and b) show that, within the precentral motor area, there are cells which receive afferent proprioceptive input. When stimulated electrically these same cells produce isolated peripheral muscle contractions. These cells may receive information identical to that transmitted to the primary somatosensory cortex which may subserve positive feedback or precentral cortical reflexes such as contact placing. Elimination of such feedback has been shown to affect the output of cells in the motor cortex (Lewis et al., 1971).

Single cell studies of the cortex (Schneider, 1972; Dreyer et al., 1974) reveal the presence of deep-submodality receiving cells from the hindlimb in Brodmann's areas 1 and 2 (postcentral cytoarchitectural areas known to receive such a proprioceptive population). However, there is a reduction of the numbers of proprioceptive neurones from the forelimbs of animals with high cervical dorsal funicular tractotomies, and from the hindlimbs of animals with damage laterally in the dorsal spinocerebellar tract or with unilateral hemisections. These data support the interpretation that the segregation of exteroceptive and proprioceptive afferent fibres within the spinal 
cord (Whitsel et al., 1969, 1970) can account for the selective diminution of proprioceptive or exteroceptive cells from the cortical population when tractotomies transect these afferent fibres after they have been separated. Thus, spinal cord fibres sorting can be shown to play an important role in the determination of the body representation in the primary somatosensory cortex (Whitsel et al., 1972). Such fibre sorting contributes to the evaluation of the rostro-caudal level and extent of spinal cord lesions.

It should be noted that the assessment of proprioceptive losses may involve a number of related, but different qualities. For example, Ferraro and Barrera (1934) used the failure of slow movements of any limb to evoke eye movements in their fourth cervical preparation to attribute loss of proprioception to their dorsal funiculus interruption. We find this phenomenon also, but are not willing to say it implies complete absence of proprioceptive sense. These authors also noted the prominent dorsal placing of the distal limbs of such animals, and in one case used it to assign proprioceptive loss to the hindlimb. Our electrophysiological results indicate that the marked impairment of cutaneous sensation on the volar surface of both distal limbs, observed after dorsal funiculus transection above the entry of afferent fibres from those limbs, could probably result in this unusual posture, the feedback necessary for spatial position sense at rest being obtainable only from the remaining innervation of the foot and hand. Thus, an exteroceptive rather than a proprioceptive loss may be the basis for this observation. Schiff, in 1857 , made a similar claim (cf. Ferraro and Barrera, 1934). Gilman and Denny-Brown (1966) cite catatonic postures as evidence of loss of proprioception in animals with a high cervical dorsal funiculus lesion. While we have also observed this on occasion, the ability to use the hindlimbs without visual guidance in such animals both in walking and climbing, and in directing movements in space, indicate to us at least some preservation of proprioceptive function. Agreement on this seems to come from other dorsal funiculus tractotomy studies (Christiansen, 1966) in which neurological testing was also used.

We believe that the concentration of exteroceptive afferent fibres within the dorsal funiculus confers upon it quantitatively the largest role in conveying information useful in tactile exploration and manipulation through the macaque spinal cord. While we cannot draw the conclusion that our hair movement and tap tests of animals with transection of the dorsal funiculus demonstrated lack of sensation, we do believe that inattention or diminished sensitivity is indicated. This conviction is supported by the radical diminution in hair and touch related single cells encountered in microelectrode penetrations of the somatosensory cortex (Schneider, 1972; Dreyer et al., 1974). This diminution is significantly more extensive when the population of tactile receptors of the glabrous skin of the fore and hindlimb is considered (Schneider, 1972; Dreyer et al., 1974). In the macaque, this refers to the palm and sole. The population of tactile receptors on the palm also supplies the largest input to those cells of the hand represented in the motor cortex which produce flexion of the fingers (Asanuma and Rosen, 1972b). These data suggest critical roles for such receptors in tactile exploration, manipulation, and grasping. This is not to say that these functions must be exclusively subserved by this receptor population. In the macaque, there is evidence that other pathways can be used in tasks involving these areas (Kuhn, 1949; Schwartz et al., 1972; Vierck, 1973). Even less can we conclude that other body areas are the sole domain of one afferent system (Mettler and Liss, 1959; Christiansen, 1966; Norrsell, 1966; Kitai and Weinberg, 1968; Schwartzman and Bogdonoff, 1968, 1969; Dobry and Casey, 1972; Eidelberg and Woodbury, 1972; Mann et al., 1972; Schwartz et al., 1972; Liebman and Levitt, 1973). However, it has been demonstrated (Ferraro and Barrera, 1934; Gilman and Denny-Brown, 1966; Wall, 1970; Dubrovsky et al., 1971; Melzack and Bridges, 1971; Schneider, 1972) that the quality of behaviour which uses these receptors changes after the elimination of dorsal funiculus pathways, and even when behavioural testing is included the disturbances are nonetheless present.

With regard to the possibility that inattention is the result of a dorsal funiculus lesion, Wall (1970) presented such a hypothesis of dorsal funiculus function, especially in relation to the lack of touch deficits in human subjects with high cervical and thoracic spinal cord lesions (Boshes and Padberg, 1953). In a retest of one of these patients, Wall was able to demonstrate a change in the quality of the remaining touch in the direction of being less frequently felt during concomitant distraction of the patient by vibratory sensation, or by having him read aloud (Wall, 1970). Electrophysiological results (Schneider, 1972; Dreyer et al., 1974) indicate that many of the single neurones which are encountered in the somatosensory cortex after posterior white column transection show a rapid habituation, and require higher stimulus intensities to be excited. Such units, if 
they are characteristic of the remaining form of sensation in the patients, could account for their lack of sensitivity to one form of stimulation during simultaneous application of vibratory stimulation. Oscillatory stimulation could be like white noise, masking sporadic transmission from another somatic source.

Another question is whether the size of the cage in which the animal is confined after surgery determines the amount of recovery of function. We believe that residual postural and motor deficiencies at one month after surgery may be affected by cage size, but only to the extent that free roaming or colony caged animals have a greater opportunity to develop strategies to cope with their deficits. Such a strategy was reported (Goldberger, 1972) when tactile evasion was enhanced to enable a macaque with a lesion of Brodmann's area 6 of the cerebral cortex to overcome the pathological grasp reflex. These strategies may make the animal appear grossly to be without deficits which subtler testing would reveal.

A discussion of grasp responses must heed the differences in type and in nomenclature which have been used by various authors. The important distinction is that in ascending order of complexity lie the coarse grasp or traction reaction, the true grasp reflex, and instinctive grasping (Denny-Brown and Chambers, 1958; Twitchell, 1965). This last named reaction is a contactual orienting reaction whose voluntary performance was called spontaneous grip or spontaneous grasp by Ferraro and Barrera (1934).

The concentration of distal afferent fibres related to grasping in the dorsal funiculus causes both devastating loss of grasp behaviour with total tractotomies and resilience of it with partial sparing. Our complete dorsal funiculus lesions were sufficient to abolish the hindlimb true grasp reflex. Gilman and Denny-Brown (1966), who tested this reflex, found a brisk grasp reflex on the hindlimb in only one of their experimental animals (DC1) which had a bilateral high cervical dorsal funiculus section, but not on the other four animals with similar lesions (DC4, DC14, DC15, and DC46). Only coarse grasp reactions were elicited in these latter animals. The occurrence of the true grasp reflex in the lone animal may have resulted from the sparing of small bundles of dorsal funiculus fibres by the lesion. Such sparing appears to have resulted in the retention of this reflex even though diminished by our animals DCX: 9 and DCX: 15. However, the lesion in the one animal mentioned above does seem complete; thus, we are at a loss to explain this result.
Nevertheless, we interpret our data to indicate that the true grasp reflex depends on the dorsal funiculus.

Dorsal funiculus lesions were also sufficient to replace forelimb grasp reflexes with active avoidance responses. This occurred even when the lesions were below the brachial plexus. Hindlimb tactile avoidance was seen early in the postoperative course with many animals, but generally disappeared or was greatly diminished by the end of the first postoperative month. Gilman and Denny-Brown (1966) found such avoiding on the hindlimb but not the forelimb in one animal (DC1) and found coarse avoiding in the forelimbs of two (DC5 and DC15). A hyperaesthesia following primary somatosensory cortex ablations in macaques is reported (Schwartzman and Semmes, 1971), which we believe is identical to the phenomenon referred to by others (DennyBrown and Chambers, 1958) as release of tactile avoiding, and which occurs with more extensive postcentral cortical excisions in macaques. Thus, the limited cortical ablations will produce results similar to those which dorsal funiculus lesions create in forelimbs and hindlimbs at different postoperative times. It would seem that a tonic input from the dorsal funiculus to the postcentral cortex is necessary to maintain normal tactile reactivity.

Instinctive grasping, encompassing a higher level of complexity, is not preserved in the hindlimb by partial sparing of the dorsal funiculus (DCX: 8, 9, 15), but a greater amount of sparing caused by the same lesion (DCX: 9) being just rostral to mid-plexus level, fails to abolish this response in the forelimb. Lesions above the brachial plexus do abolish this response in the forelimb (DCX: 10, 11). Ferraro and Barrera (1934) were only able to get crude spontaneous grasping after one month in any limb of an animal with a midcervical, dorsal funiculus lesion (CER80) or in the hindlimb of a midthoracic, dorsal funiculus lesioned animal (CER135). The dorsolateral funiculus lesions alone yielded no effect on this response in either limb in our studies. Identical results were obtained by Gilman and Denny-Brown (1966) with a similar lesion. Ferraro and Barrera (1934) described the deficits in spontaneous grasping common to animals with a dorsal funiculus lesion. In an interesting observation they relate that animals with such lesions try to grip things with their entire limb rather than just its distal part. We believe this perceptive observation can be explained by the relative preservation of the proximal limb sensory input which we see in our electrophysiological work (Schneider, 1972; 
Dreyer et al., 1974). These results may also explain the necessity for using broad areas of cutaneous contact with the limbs in producing tactile placing and traction responses as described by Gilman and Denny-Brown (1966), and the findings of Vierck (personal communication) that distal forelimb deficits in proprioception are more marked than proximal deficits in macaques performing behavioural tasks after posterior whitc column tractotomies.

The existence of a sorting process and its consequences at levels rostral to the spinal cord introduce a new dimension into the analysis of dorsal funiculus studies. For example, Melzack and Bridges (1971) found that, with motor performance in cats, there was less drastic interference by lesions solely in the nuclei of the dorsal funiculus than with those which were made only in the high cervical dorsal funiculus. They regarded this as a paradoxical result. Since their lesions did not extend into an area of the nuclei which has been shown to receive a large number of proprioceptive afferent fibres (Kuhn, 1949; Gordon and Paine, 1960; Gordon and Seed, 1961; Perl et al., 1962; Gordon and Jukes, 1964; Biedenbach, 1972), that is, rostral to the obex, they may have spared an important fraction of proprioceptive fibres. It would thus become extremely important to delimit the precise caudal extent of each of their lesions since the less caudal a lesion is, the more fibres can be spared by sorting. Since dorsal funiculus nucleus lesions require a more rostral central nervous system exposure, the chances are that more sparing would have occurred in these animals than in those with dorsal funiculus lesions alone, and the fibre sorting process could explain their paradoxical results.

The effect of dorsal funiculus lesions on the behaviour of the animal derives from the limitation of his ability to receive mechanoreceptive information, especially from the distal, volar receptors most involved in palpatory input. Tactual guidance is necessary in fine manipulative tasks where vision is excluded (Brinkman and Kuypers, 1972), and its sudden loss cannot be immediately compensated by visual input alone. Manipulations where spared sensory input may be used-for example, vision, facial somatosensation, crude hand and foot dorsum somatosensation-like those involving feeding and grooming, tend to show quicker recovery, but a return to the status before the transection does not occur within a month. Recent behavioural data have shown that when selective ablations are made of the anterior and posterior postcentral gyrus (Semmes and Porter, 1972), or of Brodmann's cytoarchitectonic areas
3, 1, and 2 (Randolph and Semmes, 1974), the region which is the primary central terminus of impulses travelling along dorsal funiculus fibres, there are differentiable, discriminative, behavioural deficits which last for months. Again, the receptor information which permits discrimination by palpation seems to be disturbed, and in addition, the disturbance varies with the cytoarchitectural area involved.

The picture that emerges is that submodalities of mechanoreceptive somatosensation, and perhaps types of receptor information within submodalities, are segregated as they ascend from caudal to cephalic levels of the nervous system so as to form somatosensory subunits in the primary cortical receiving area (Mountcastle and Powell, 1959a, b; Powell and Mountcastle, 1959a, b; Whitsel et al., 1972). The sorting process that takes place in the dorsal funiculus contributes to this segregation (Whitsel et al., 1969; Whitsel et al., 1970). This sorting, and the existence of alternative somatosensory pathways, however different in the manner in which they handle the stimulus information, allow for the development of strategies to assist the recovery of function assessed by many experiments after dorsal funiculus lesions in animals (Devito and Ruch, 1956; Mettler and Liss, 1959; Christiansen, 1966; Levitt and Schwartzman, 1966; Vierck, 1966; Kitai and Weinberg, 1968; Schwartzman and Bogdonoff, 1968, 1969; Dobry and Casey, 1972; Eidelberg and Woodbury, 1972; Mann et al., 1972; Schneider, 1972; Schwartz et al., 1972; Liebman and Levitt, 1973). Neurological observations do not permit the same statement to be made about humans, because those studies which report absence of deficits after dorsal funiculus tractotomy lack the necessary histological confirmation (Rabiner and Browder, 1948; Boshes and Padberg, 1953; Cook and Browder, 1965). Also, neurological examinations in humans continue to implicate the dorsal funiculus in postural sensation (Rose and Mountcastle, 1959; Poggio and Mountcastle, 1960). Whether differences related to assessment procedures may account for these results in humans is not yet known. However attractive the new hypotheses of dorsal funiculus function may be in offering explanations of the physiological role of the posterior white columns, we suggest that further physiological and behavioural evidence is required before conclusions can be drawn about the function of the dorsal funiculus. While we believe that our re-sorting hypothesis is correct in monkey, and may apply to man, we admit that it goes beyond existing evidence. We are currently recording the appropriate clinical information in patients. 


\section{References}

Albe-Fessard, Denise (1967). Organization of somatic central projections. In Contributions to Sensory Physiology. Vol. 2. Edited by W. D. Neff. Academic Press: New York.

Andersson, S. A., Landgren, S., and Wolsk, D. (1965). The thalamic relay and cortical projection of group I muscle afferents from the forelimb of the cat. Journal of Physiology, 183, 576-591.

Asanuma, H., and Rosén, I. (1972a). Functional role of afferent inputs to the monkey motor cortex. Brain Research. 40, 3-5.

Asanuma. H., and Rosén, I. (1972b). Topographical organization of cortical efferent zones projecting to distal forelimb muscles in the monkey. Experimental Brain Research, 14, 243-256.

Biedenbach, Maria A. (1972). Cell density and regional distribution of cell types in the cuneate nucleus of the Rhesus monkey. Brain Research, 45, 1-14.

Boshes, B., and Padberg, F. (1953). Studies on the cervical spinal cord of man. Neurological Society of Minneapolis, 3, 90-101.

Bowsher, D. (1965). The anatomophysiological basis of somatosensory discrimination. International Review of Neurobiology, 8, 35-75.

Brinkman. J., and Kuypers, H. G. J. M. (1972). Splitbrain monkeys: cerebral control of ipsilateral and contralateral arm, hand, and finger movements. Science, 176, 536-539.

Burgess, P. R., and Clark, F. J. (1969). Dorsal column projection of fibres from the cat knee joint. Journal of Physiology, 203, 301-315.

Carlson, Kristen R. (1972). A temporary restraint chair for monkeys. Physiology and Behavior, 9, 493-494.

Christiansen, J. (1966). Neurological observations of macaques with spinal cord lesions. Anatomical Record, 154, 330.

Cook, A. W.. and Browder, E. J. (1965). Functions of posterior columns in man. Archives of Neurology (Chicago), 12, 72-79.

Denny-Brown. D.. and Chambers, R. A. (1958). The parietal lobe and behavior. In The Brain and Human Bêhavior. Edited by H. C. Solomon, S. Cobb, and W. Penfield. Williams and Wilkins: Baltimore.

Devito, J. L., and Ruch, T. C. (1956). Central pathways subserving weight discrimination in monkeys. Federation Proceedings, 15, 48-49.

Dobry. P. J. K., and Casey, K. L. (1972). Roughness discrimination in cats with dorsal column lesions. Brain Research, 44, 385-397.

Dreyer, D. A.. Schneider, R. J., Metz. C. B., and Whitsel, B. L. (1974). Differential contributions of spinal pathways to body representation on the postcentral gyrus. Journal of Neurophysiology, 37, 119-145.

Dubrovsky. B., Davelaar, E., and Garcia-Rill, R. (1971). The role of dorsal columns in serial order acts. Experimental Neurology, 33, 93-102.

Eidelberg, E.. and Woodbury, C. M. (1972). Apparent redundancy in the somatosensory system in monkeys. Experimental Neurology, 37, 573-581.

Ferraro, A.. and Barrera, S. E. (1934). Effects of experimental lesions of the posterior columns in Macacus rhesus monkeys. Brain, 57, 307-332.

Gilman, S., and Denny-Brown. D. (1966). Disorders of movement and behaviour following dorsal column lesions. Brain, 89, 397-418.

Glees, P., and Soler, J. (1951). Fibre content of the posterior column and synaptic connections of nucleus gracilis. Zeitschrift für Zellforschung. 36, $381-400$.

Goldberger, M. E. (1972). Restitution of function in the CNS: the pathologic grasp in Macaca mulatta. Experimental Brain Research, 15, 79-96.

Gordon, G., and Jukes, M. G. M. (1964). Dual organization of the exteroceptive components of the cat's gracile nucleus. Journal of Physiology, 173, 263-290.

Gordon, G., and Paine. C. H. (1960). Functional organization in the nucleus gracilis of the cat. Journal of Physiology, 15, 331-349.

Gordon, G., and Seed, W. A. (1961). An investigation of nucleus gracilis of the cat by antidromic stimulation. Journal of Physiology, 155, 589-601.

Ha, H. (1971). Cervicothalamic tract in the Rhesus monkey. Experimental Neurology, 33, 205-212.

Ha, H., and Morin, F. (1964). Comparative anatomical observations of the cervical nucleus, $N$. cervicalis lateralis, of some primates. Anatomical Record, 148, 374-375.

Hand, P. J. (1966). Lumbosacral dorsal root terminations in the nucleus gracilis of the cat. Some observations on terminal degeneration in other medullary sensory nuclei. Journal of Comparative Neurology, 126, 137-156.

Iggo, A. (1963). An electrophysiological analysis of afferent fibres in primate skin. Acta Neurovegetativa, 24, 226-240.

Jasper, H., and Bertrand, G. (1964). Stereotaxic microelectrcde studies of single thalamic cells and fibres in patients with dyskinesia. Transactions of the American Neurological Association. 89, 79-82.

Kitai, S. T., and Weinberg, J. (1968). Tactile discrimination study of the dorsal column-medial lemniscal and spinocervico-thalamic tract in cats. Experimental Brain Research, 6, 234-246.

Kuhn, R. A. (1949). Topographical pattern of cutaneous sensibility in the dorsal column nuclei of the cat. Transactions of the American Neurological Association, 74, 227-230.

Kuypers, H. G. J. M., and Tuerk, J. D. (1964). The distribution of the cortical fibres within the nuclei cuneatus and gracilis in the cat. Journal of Anatomy, 98, 143-162.

Landgren, A., Nordwall, A., and Wengstrom, C. (1965). The location of the thalamic relay in the spino-cervico-lemniscal path. Acta Physiologica Scandinavica, 65, 164-175.

Levitt, M., and Schwartzman, R. (1966). Spinal sensory tracts and two-point tactile sensitivity. Anatomical Record, 154, 476.

Lewis, M. McD., Porter, P., and Horne, M. (1971). 
The effects of impairment of afferent feedback from the moving limb on the natural activities of neurones in the precentral gyrus of conscious monkeys: A preliminary investigation. Brain Research, 32, 467-473.

Liebman, R. S., Levitt, M. (1973). Position sense after combined spinal tractotomies and cerebellectomies in macaques. Experimental Neurology, 40, 170-182.

Lundberg, A., and Norrsell, U. (1960). Spinal afferent pathways of the tactile placing reaction. Experientia, 16, 123.

Mallart, A. (1964). Projection des afférences musculaires de la patte antérieure au niveau du thalamus chez le chat. Comptes Rendus Hebdomadaires des Séances (Paris), 259, 1215-1218.

Mann, M. D., Kasprzak, H., Hiltz, F. L., and Tapper, D. N. (1972). Activity in single cutaneous afferents: spinal pathways and cortical evoked potentials. Brain Research, 39, 61-70.

Melzack, R., and Bridges, J. A. (1971). Dorsal column contributions to motor behavior. Experimental Neurology, 33, 53-68.

Mettler, R. A., and Liss, H. (1959). Functional recovery in primates after large subtotal spinal cord lesions. Journal of Neuropathology and Experimental Neurology, 18, 509-516.

Mountcastle, V. B., and Powell, T. P. S. (1959a). Central nervous mechanisms subserving position sense and kinesthesis. Bulletin of the Johns Hopkins Hospital, 105, 163-200.

Mountcastle, V. B., and Powell, T. P. S. (1959b). Neural mechanisms subserving cutaneous sensibility with special reference to the role of afferent inhibition in sensory perception and discrimination. Bulletin of the Johns Hopkins Hospital, 105, 201232.

Mountcastle, V. B., and Smith, I. D. (1968). Central nervous mechanisms in sensation. In Medical Physiology. Vol.2. Edited by V. Mountcastle. C. V. Mosby: St. Louis.

Norrsell, U. (1966). The spinal afferent pathways of conditional reflexes to cutaneous stimuli in the dog. Experimental Brain Research, 2, 269-282.

Norrsell, U., and Voorhoeve, P. (1962). Tactile pathways from the hindlimb to the cerebral cortex in cat. Acta Physiologica Scandinavica, 54, 9-17.

Perl, E. R., Whitlock, D. G., and Gentry, J. R. (1962). Cutaneous projection to second order neurones of the dorsal column system. Journal of Neurophysiology, 25, 337-358.

Petit, D., and Burgess, P. R. (1968). Dorsal column projection of receptors in cat hairy skin supplied by myelinated fibers. Journal of Neurophysiology, 31, 849-855.

Poggio, G. F., and Mountcastle, V. B. (1960). A study of the functional contributions of the lemniscal and spinothalamic systems to somatic sensibility. Central nervous mechanisms in pain. Bulletin of the Johns Hopkins Hospital, 106, 266316.

Powell, T. P. S., and Mountcastle, V. B. (1959a).
The cytoarchitecture of the postcentral gyrus of the monkey Macaca mulatta. Bulletin of the Johns Hopkins Hospital, 105, 108-132.

Powell, T. P. S., and Mountcastle, V. B. (1959b). Some aspects of the functional organization of the cortex of the postcentral gyrus of the monkey: a correlation of findings obtained in a single unit analysis with cytoarchitecture. Bulletin of the Johns Hopkins Hospital, 105, 133-162.

Rabiner, A. M., and Browder, E. J. (1948). Concerning conduction of touch and deep sensibilities through the spinal cord. Transactions of the American Neurological Association, 73, 137.

Randolph, M., and Semmes, J. (1974). Behavioral consequences of selective subtotal ablations in the postcentral gyrus of Macaca mulatta. Brain Research, 70, 55-70.

Rose, J., and Mountcastle, V. B. (1959). Touch and kinesthesis. In Handbook of Physiology, Section 1, Neurophysiology, Vol. 1. Edited by J. Field, H. W. Magoun, and V. E. Hall. American Physiological Society: Washington, DC.

Schneider, R. J. (1972). The effects of lesions of the posterior funiculi of Macaca mulatta. PhD Dissertation, University of Pittsburgh.

Schwartz, A. S., Eidelberg, E., Marchok, P., and Azulay, A. (1972). Tactile discrimination in the monkey after section of the dorsal funiculus and lateral lemniscus. Experimental Neurology, 20, 4351 .

Schwartzman, R. J., and Bogdonoff, M. D. (1968). Behavioral and anatomical analysis of vibration sensibility. Experimental Neurology, 20, 43-51.

Schwartzman, R. J., and Bogdonoff, M. D. (1969). Proprioception and vibration sensibility discrimination in the absence of the posterior columns. Archives of Neurology (Chicago), 20, 349-353.

Schwartzman, R. J., and Semmes, J. (1971). The sensory cortex and tactile sensitivity. Experimental Neurology, 33, 147-158.

Semmes, J. (1969). Protopathic and epicritic sensation: a re-appraisal. In Contributions to Clinical Neuropsychology. Edited by A. L. Benton. Aldine Publishing: Chicago.

Semmes, J., and Porter, L. (1972). A comparison of pre-central and postcentral cortical lesions on somatosensory discrimination in the monkey. Cortex, 8, 249-264.

Twitchell, T. E. (1965). The automatic grasping responses of infants. Neuropsychologia, 3, 247-259.

Vierck, C. J. (1966). Spinal pathways mediating limb position sense. Anatomical Record, 154, 437.

Vierck, C. J. (1973). Alterations of spatio-tactile discrimination after lesions of primate spinal cord. Brain Research, 58, 69-79.

Wall, P. D. (1970). The sensory and motor role of impulses travelling in the dorsal columns towards cerebral cortex. Brain, 93, 505-524.

Walshe, Sir Francis. (1970). Diseases of the Nervous System. Williams and Wilkins: Baltimore.

Whitsel, B. L., Petrucelli, L. M., and Sapiro, G. (1969). Modality representation in the lumbar and 
cervical fasciculus gracilis of squirrel monkeys. Brain Research, 15, 67-78.

Whitsel, B. L., Petrucelli, L. M., Sapiro, G., and Ha, H. (1970). Fiber sorting in the fasciculus gracilis of squirrel monkeys. Experimental Neurology, 29, 227242.

Whitsel, B. L., Petrucelli, L. M., Ha, H., and Dreyer,
D. A. (1972). The resorting of spinal afferents as antecedent to the body representation in the postcentral gyrus. Brain Behavior and Evolution, 5, 303-341.

Woolsey, C. N., and Bard, P. (1963). Cortical control of hopping and placing reactions in Macaca mulatta. A merican Journal of Physiology, 116, 165. 